\title{
Autonomic Approach to Planning and Scheduling in Networked Small Factories
}

\author{
Flavio Bonfatti ${ }^{1}$, Luca Martinelli ${ }^{1}$, and Paola Daniela Monari ${ }^{2}$ \\ ${ }^{1}$ DII, University of Modena and Reggio Emilia,Via Vignolese 905, \\ 41125 Modena, Italy \\ ${ }^{2}$ SATA srl,Via Notari 103, 41126 Modena, Italy \\ flavio.bonfatti@unimore.it, p.monari@satanet.it, \\ luca.martinelli@unimore.it
}

\begin{abstract}
The paper considers the complex behavior of a small company in a business ecosystem, time by time leader or supplier or both in one or more supply chains, and points out the data exchange and processing problems it must face for reacting fast to customer requests. Then the paper suggests that in order to meet these requirements it is convenient to provide every company with proper automatic functions, covering its distributed planning, resource scheduling and exception handling needs, whose policies can be fully customised by the company itself to mirror its specific intentions: in short, a practical implementation of the autonomic model.
\end{abstract}

Keywords: autonomic approach, small company, distributed planning, resource scheduling, self-configuration, self-optimization, self-healing.

\section{Introduction}

The paper aims at showing how the autonomic approach is suited to solve the planning and scheduling problem in factories constituted by networked small companies. The focus is put on small companies (less than 50 employees) since they represent the great majority of companies and in spite of this they are normally disregarded by the offer of advanced yet affordable ICT solutions [1].

Networked small factories are normally organised into regional and/or sectoral business ecosystems (or breeding environments), thus sharing habits, tacit knowledge and informal relations. In general they follow co-evolutionary models showing the autonomous development of the involved parties [2] and need communication and collaboration supports to efficiently share their production resources [3].

Small distributed factories reach high levels of effectiveness. They innovate products by adapting fast to the changing market demand, their products are of good quality, and a significant percentage of their production is sold in other countries thus showing the attitude to be active in the global market. However, while effectiveness is already there what is actually missing is a reasonable level of efficiency.

Efficiency relates to a number of aspects such as (a) fast response time to requests for quotation and customer orders, (b) ability to establish more dynamic relations with suppliers and subcontractors, (c) better allocation of internal resources and monitoring 
of their behavior, (d) better logistics to minimise transport costs of worked materials, (e) strict collaboration with partners to damp down exceptions and perturbations. In short: higher productivity and competitiveness.

The vision is for a manufacturing environment where every small factory has access to a suite of autonomic ICT functions relating it, on the one side, with its customers and, on the other side, with its suppliers and subcontractors, and supporting both distributed planning and scheduling of internal resources.

While processes are completely automated, each company remains autonomous and has the right to customize the policies of its automatic behavior. The solution must be scalable, easy to access and use, actually customisable, cheap and not diverting company resources from the core business.

The following of this paper is made of four main sections. Section 2 briefly presents the collaboration scenario constituting the operational context for the proposed approach. Section 3 recalls the functions of the autonomic model and maps them onto the main collaboration steps. Section 4 examines the recursive network planning procedure needed to instantiate the supply chain for the fulfillment of a customer order. Finally, Section 5 examines the internal resource scheduling task that every supplier must carry out to answer the supply chain leader invitation.

\section{Collaboration Scenario}

Imagine a business ecosystem grouping small companies that are specialized in complementary manufacturing activities as producers, subcontractors or suppliers and are used to participate in several supply chains. Imagine now that one of them is reached by a customer order (similarly for a request for quotation). This event will trigger a succession of consequent actions that is worth recalling here briefly:

- The receiving company must activate its supply chain, if any, as leader (see Figure 1 left). To this purpose it will carry out a planning activity to decide which tasks are better performed internally, which are conveniently assigned to partners and which are the preferred partners in those specific cases.

- According to this plan the leader company will communicate the assigned task to each of the involved partners. If one partner is leading in turn a supply chain it will carry out a similar planning activity with the involvement of further partners to fulfill the received task (see Figure 1 right).

- The result of this distributed planning process is the configuration of the working supply chain for that customer order. The configuration will be static if partners are predefined for every task to assign; it will be dynamic if partners are selected within constellations of competing companies [4].

- As last step each involved company, including leaders, will undertake a scheduling activity for the tasks to perform internally in order to allocate and book the required resources on routings and phases. This is also the step for each company to determine lead time and cost of the assigned task.

The propagation of the distributed planning process across the ecosystem is intended to select the suited supply chain members to fulfill that specific customer order and commit them to participate in the forthcoming execution of the planned and scheduled tasks. The process will end when all the devised activities will be successfully 

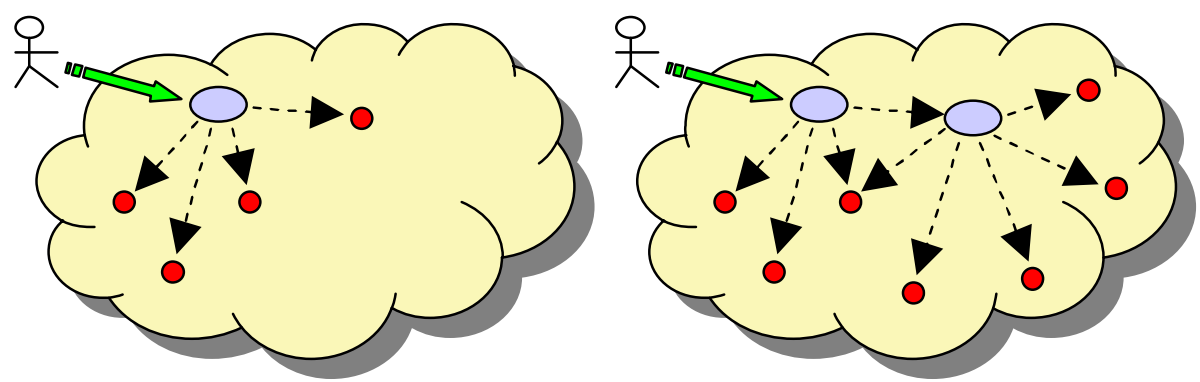

Fig. 1. A company in the business ecosystem is reached by a customer order. As a consequence it activates its supply chain (left) by sending the proper requests to its members. Every member with its own supply chain will activate it in turn (right).

covered and this will produce proper changes on the work calendars of the allocated internal resources at each selected partner.

Later, during order execution, the distributed plan will be put in action and the tasks progressively carried out by the selected companies. Transports will be performed whenever the materials need to be worked in different locations. Should a task be affected by problems (delay, loss of materials) this will trigger a replanning effort for damping down the perturbation while propagating it to the other partners.

\section{The Autonomic Model}

The Autonomic Computing Initiative (ACI) was launched by IBM in 2001 [5] as a self-management capability for facing the rapidly growing complexity of distributed computing. The essence of autonomic computing is automating low-level management tasks. The adjective is taken from the autonomic nervous system of the human body that controls important functions without any conscious intervention.

More recently, the Autonomic Network Architecture (ANA) integrated project cofunded by the European Commission [6] moved the focus to network organization based on the application of the autonomic principle. The ultimate goal was the dynamic adaptation and re-organization of the network according to the working, economical and social needs of the users. The project results confirmed the application potential of such novel network architecture.

In an autonomic system the human operator takes on a new role: he does not control the system directly, instead he defines general policies and rules that serve as an input for the self-management process. For this process the ACI defines the following four functional areas:

- Self-Configuration. This means automatic configuration of a subset of nodes in a certain zone of the network. This function is then strongly localized and includes the addition of nodes as well as the creation and cancellation of relations between nodes in order to meet the defined requirements.

- Self-Optimization. This means automatic allocation of resources in the single node to ensure an optimal functioning with respect its expected behaviour. The 
optimization algorithm is driven by performance objectives set during the selfconfiguration phase and by policies set by the node responsible.

- Self-Healing. This means automatic discovery and correction of faults. Selfhealing methods are triggered by events or exceptions occurring in a single node or by changing requirements. Normally self-healing propagates through the network and impacts on configuration and optimization.

- Self-Protection. This means proactive identification of and protection from defaulting conditions or intentional attacks. Since in networked small factories the main cause of faults is the negative behaviour of certain nodes, self-protection is mostly based on evaluation of past performances.

The adaptation of the autonomic model to the approach proposed by this paper establishes the following relations: self-configuration for distributed planning, selfoptimization for internal resource scheduling, self-healing for (distributed and internal) exception handling. Self-protection affects the previous functions with performance indicators derived by off-line functions.

Every company is subject in principle to a double autonomic process, respectively as leader of the supply chain and as subcontractor (Figure 2). If leader it will be provided with self-configuration and self-healing functions allowing automatic reaction to customer orders and automatic propagation of purchase orders to suppliers. If supplier, possibly of itself, it will automatically manage the internal scheduling phase and face possible exception coming from the shop-floor.

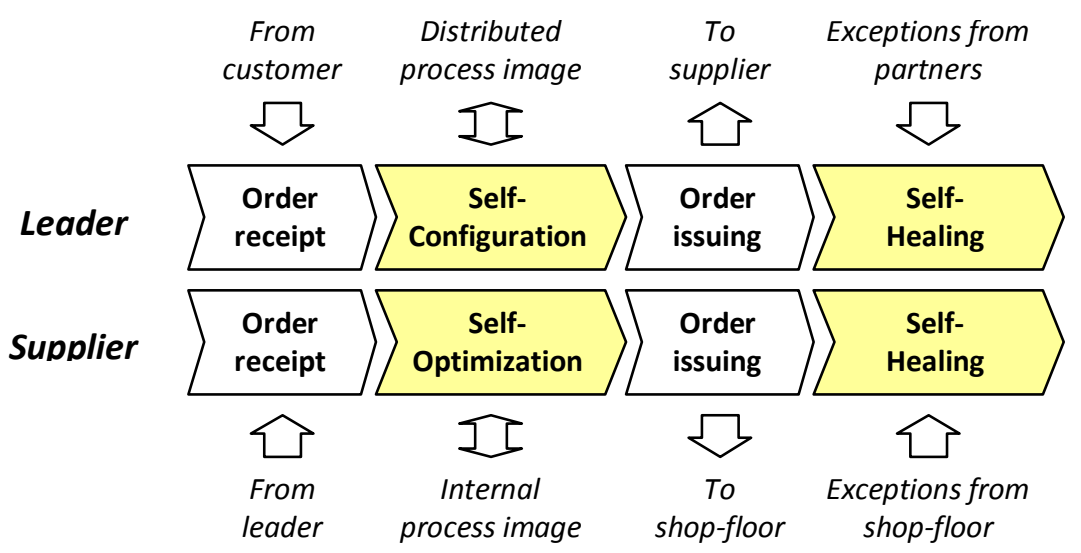

Fig. 2. The full autonomic process is triggered by the receipt of a customer order and leads to planning the supply chain, issuing purchase orders and scheduling the internal resources.

\section{Distributed Planning}

Instantiating the supply chain to fulfill the operational needs triggered by a customer request is a complex task to which much research effort has been devoted in the last years from the methodological [7] and implementation [8] viewpoints. Our approach exploits the autonomic concept and model with the ultimate aim of relieving small companies from repeatedly planning tasks and negotiations with partners. 
To this purpose it is worth analyzing what happens when a customer order for the supply of a certain quantity of product $X$ reaches that company. In general, the process $\mathrm{P}(\mathrm{X})$ for manufacturing product $\mathrm{X}$ includes a number of activities linked to each other by sequence, parallelism and alternative relations (Figure 3).

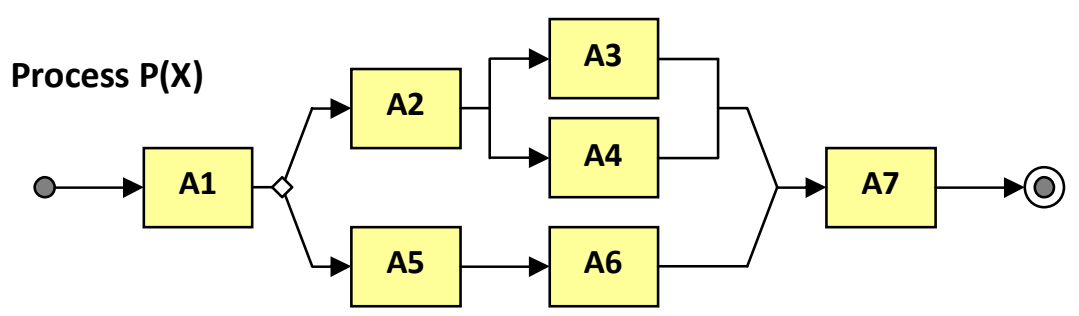

Fig. 3. Example of distributed manufacturing process. It includes seven activities where A3 and A4 are executed in parallel while the A2 and A5 braches correspond to alternative paths.

- Scan process $\mathrm{P}(\mathrm{X})$ left to right and identify A1 as first activity. Since A1 devises one or more candidate suppliers $\mathrm{Sj}$, possibly including the "make" option, each of them will be informed of the possible involvement in A1 starting from time T1 and asked to foresee its respective lead time $\mathrm{T} 1 \mathrm{j}$ and cost $\mathrm{C} 1 \mathrm{j}$.

- Consider now the upper branch and its first activity A2. Having in turn one or more candidate suppliers $\mathrm{Sk}$, every pair $(\mathrm{Sj}, \mathrm{Sk})$ must be considered in order to estimate time Tjk and cost Cjk for moving materials (if any) from the one to the other. The starting time $\mathrm{T} 2$ for activity $\mathrm{A} 2$ is then given by $\mathrm{T} 1+\mathrm{T} 1 \mathrm{j}+\mathrm{Tjk}$.

- With the following parallel activities A3 and A4 the number of combinations increases further but the procedure does not change: each candidate supplier of every activity is informed of the estimated start time for that activity and is asked to foresee time and cost of its possible contribution to process $\mathrm{P}(\mathrm{X})$.

- At the end of this procedure the algorithm has available a complete estimation of all the feasible process instantiations for that order, and detailed measures of intermediate and final lead times and costs. It can then choose the best one according to the established policy (e.g. the fastest, the cheapest, etc.).

The algorithm can be simplified to reduce the number of combinations to consider. To this purpose the policy could include some heuristics for the early selection of candidate suppliers or choice among alternative paths. In any case, reaching the optimal or suboptimal solution requires an intense message traffic with candidate suppliers that is hardly managed in a small company by means of not automatic tools.

This problem is technically overcome by providing every company with automatic self-configuration and self-healing functions based on the described planning algorithm. The human intervention remains to identify interesting partners and define the policy that such a company intends to autonomously apply for driving the process instantiation phase and the exception handling phase. The benefits are important:

- No resource must be diverted from the company core business to manage real-time interactions with partners. 
- The final response to the customer and the assignment of tasks to the selected partners are fast and accurate.

- The reaction to exceptions is also computed accurately, in a very short time and with minimal consequences.

It is worth observing that these benefits are particularly important to small companies, while larger ones are normally provided with resources letting them manage more easily the process configuration and re-planning phases. In other words, the smaller is a company the greater can be its advantage in adopting a full autonomic solution. Of course, other problems arise e.g. the need to build confidence towards an approach that apparently subtracts the operation control to them.

\section{Resource Scheduling}

The last critical aspect of a full autonomic solution is the support that must be assured to each small company behaving as supplier in the supply chain. To this purpose let us analyze what happens when the supply chain leader proposes to this company to perform activity $\mathrm{Ai}$ belonging to process $\mathrm{P}(\mathrm{X})$. Normally the execution model of $\mathrm{Ai}$ is represented in form of a routing made by a sequence of operational phases.

A phase is intended by the shop-floor as an individual operation to be carried out by a certain type of resource team. The resource team type is composed by a combination of primary and secondary resources (persons, machines, equipment, etc.) for each of which the company has available one or more instances. Scheduling the execution of activity $\mathrm{Ai}$ means allocating the resource instances able to perform the phases of the relative routing. More precisely:

- The routing is scanned one phase at a time. The first phase is expected to start as soon as possible after the established start time Ti for activity Ai. In practice it will start when an instance of the required resource team can be organized and allocated to it, taking into account the engagements of resource instances.

- To this purpose the work calendars of the proper resource instances are examined and one or more combinations are identified with the constraint to involve them for the estimated duration of that phase. The choice of the best combination will be based on the policy established by that company.

- After scheduling the resources allocated to the first phase it is possible to derive its cost and the delivery time of the worked materials. The latter is the reference start time for the next phase, with possible positive or negative slacks.

- At the end of the entire scheduling procedure the total activity cost and the total lead time are finally known and can be communicated to the leader company for feeding its planning decisions.

- Later, during task execution, exceptions can occur e.g. because of delayed operations, missing resource instances, loss of materials. The schedule must be modified in such a way to solve them and, if this is not sufficient, they must be communicated to the supply chain leader for higher-level intervention.

Although not so complex like the planning algorithm, the scheduling algorithm must however consider a large number of variables and conditions, and apply a policy that 
can be in turn quite complex. This calls again for an automated solution under the condition that it leaves wide margins for the definition of autonomous policies.

\section{Conclusions}

Small companies behaving as nodes of networked factories, with both leader and supplier roles, must increase their efficiency or risk to be left aside of the increasingly competitive global market. Being provided with limited resources that they cannot divert from the core business, it is fundamental for them to adopt strongly automated planning, scheduling and exception handling solutions under the condition to maintain wide margins for policy customization.

This can effectively be done by autonomic functions for dynamic configuration of the networked factory, optimization of resource allocation within every node, automation of healing reactions to exceptions, policy enrichment with node performance indicators. The paper has shown the way to realize this solution starting from an analysis of the collaboration scenario representing the intended behaviors.

The autonomic functions for a networked small factory are presently studied and developed in the frame of the EU co-funded FP7-Capacities-SME 243554 eBEST project [9] with special attention to the subcontracting sector. The new ICT solution will be subject to validation by networks established in six European regions, namely Belgium, Greece, Hungary, Italy, Slovakia and Spain.

\section{References}

1. e-Business Watch: An Economic Assessment of ICT-related Industrial Policy (2009), http: / / www. ebusinesswatch.org/studies/special_topics/2009/ICT_policy.htm

2. Dekkers, R.: Distributed Manufacturing as Co-evolutionary System. International Journal of Production Research 47(8) (2009)

3. Noori, H., Lee, W.B.: Dispersed Network Manufacturing: an Emerging Form of Collaborative Networks. In: Dekkers, R. (ed.) Dispersed Manufacturing Networks: Challenges for Research and Practice. Springer, Heidelberg (2009)

4. Camarinha-Matos, L.M., Afsarmanesh, H.: A framework for virtual organization creation in a breeding environment. Journal Annual Reviews in Control 31(1), 119-135 (2007)

5. IBM Autonomic Computing Initiative, http://www01.ibm.com/software/tivoli/autonomic/ (visited March 2010)

6. Rodriguez-Fernandez, D., et al.: Demonstrating Autonomic On-demand Content Delivery. In: Mauthe, A., Zeadally, S., Cerqueira, E., Curado, M. (eds.) FMN 2009. LNCS, vol. 5630, pp. 206-211. Springer, Heidelberg (2009)

7. Romero, D., et al.: A Virtual Breeding Environment Reference Model and its Instantiation Methodology. In: Proceedings of PRO-VE 2008, Poznan (2008)

8. Ignatiadis, I., et al.: Panda - a Virtual Breeding Environment for SMEs in the ERP/CRM Industry Using a Service Oriented Approach. World review of Science, Technology and Sustainable Development 7, 1-2 (2010)

9. The eBEST project, http://www. ebest. eu 